$$
\begin{aligned}
& \text { ANL/XFD/CP- } 90424 \\
& \text { CONF-960824-- } 2
\end{aligned}
$$

\title{
X-RAY OPTICS DEVELOPMENTS AT THE APS FOR THIRD-GENERATION SYNCHROTRON RADIATION SOURCES*
}

\author{
Dennis M. Mills \\ Experimental Facilities Division \\ Advanced Photon Source, Argonne National Laboratory \\ Argonne, IL 60439 \\ RECEIVED
NOV 211996
OSTI
}

September 1996

\begin{abstract}
The submitted manuscript has been created by the University of Chicago as Operator of Argonne National Laboratory ("Argonne") under Contract No. W-31-109-ENG-38 with the U.S. Department of Energy. The U.S. Government retains for itself, and others acting on its behalf, a paid-up, nonexclusive, irrevocable worldwide license in said article to reproduce, prepare derivative works, distribute copies to the public, and perform publicly and display publicly, by or on behalf of the Government.
\end{abstract}

INVITED TALK presented at the IUCr Synchrotron Radiation Satellite Meeting, Argonne, IL, August 4-7, 1996; to be published in the Proceedings, Journal of Synchrotron Radiation.

\footnotetext{
*This work supported by the U.S. Department of Energy, Basic Energy Sciences-Materials Sciences, under contract \#W-31-109-ENG-38.
} 


\title{
X-Ray Optics Developments at the APS \\ for Third-Generation Synchrotron \\ Radiation Sources
}

Dennis M. Mills

Advanced Photon Source

Argonne National Laboratory

9700 S. Cass Ave., Argonne, IL 60439

\begin{abstract}
High brilliance third-generation synchrotron radiation sources simultaneously provide both a need and an opportunity for the development of new x-ray optical components. The high power and power densities of the $x$-ray beams produced by insertion devices have forced researchers to consider novel, and what may seen like exotic, approaches to the mitigation of thermal distortions that can dilute the beam brilliance delivered to the experiment or next optical component. Once the power has been filtered by such high heat load optical elements, specialized components can be employed that take advantage of the high degree of brilliance. This presentation reviews the performance of optical components that have been designed, fabricated, and tested at the Advanced Photon Source, starting with high heat load components and followed by examples of several specialized devices, such as a milli-eV resolution (in-line) monochromator, a high energy $x$-ray phase retarder, and a phase zone plate with submicron focusing capability.
\end{abstract}




\section{DISCLAIMER}

This report was prepared as an account of work sponsored by an agency of the United States Government. Neither the United States Government nor any agency thereof, nor any of their employees, makes any warranty, express or implied, or assumes any legal liability or responsibility for the accuracy, completeness, or usefulness of any information, apparatus, product, or process disclosed, or represents that its use would not infringe privately owned rights. Reference herein to any specific commercial product, process, or service by trade name, trademark, manufacturer, or otherwise does not necessarily constitute or imply its endorsement, recommendation, or favoring by the United States Government or any agency thereof. The views and opinions of authors expressed herein do not necessarily state or reflect those of the United States Government or any agency thereof. 


\section{DISCLADMER}

Portions of this document may be illegible in electronic image products. Images are produced from the best available original document. 


\section{Introduction}

The third generation of synchrotron radiation sources that have recently become operational in Europe and the United States, and will soon become operational in Japan, provide simultaneously new technical challenges and scientific opportunities for the designers of $\mathbf{x}$-ray optical components. X-ray beams generated by the undulators installed in the straight sections of these new storage rings not only have very high levels of brilliance (typically greater than $10^{19}$ photons/sec-0.1\%bw-mm². $\mathrm{mrad}^{2}$ ) but also very high total power levels (kilowatts) and power densities (hundreds of watts $/ \mathrm{mm}^{2}$ ) associated with them. A summary of some of the latest experimental results that have been obtained at the Advanced Photon Source (APS) in the development and testing of both high heat load optics and novel x-ray optical components will be described here. (For a recent overview of optics development at the European Synchrotron Radiation Facility (ESRF) see Ref.1.)

\section{High Heat Load Optics}

A variety of approaches have been explored by the APS staff to mitigate the thermal distortion effects in optics exposed directly to the undulator beam. For single-crystal-monochromator crystal optics, we have had the best results with cryogenically cooled silicon and room temperature, synthetic diamond components. Neither of these two approaches are new, both having been pioneered at the ESRF [1-3]. However, because of the larger total powers and higher power densities that occur at the APS, modification of the ESRF designs were in some cases required.

Cryogenically cooled optics for use with high power synchrotron radiation beams was first suggested in 1985 for mirrors [4] and in the following year for crystal monochromators [5]. As was pointed out in those papers, the advantage of operating single-crystal-silicon optical components at cryogenic temperatures is twofold: (1) the thermal conductivity, $k$, increases by nearly a decade in going from room temperature to liquid-nitrogen temperatures while (2) the coefficient of thermal expansion, $\alpha$, decreases from its room temperature value of $2.3 \mathrm{x}$ $10^{-6} / \mathrm{K}$, going through zero at $125^{\circ} \mathrm{K}$, and then remains small and negative near the boiling temperature of liquid nitrogen - see Table I. (Liquid nitrogen is a inexpensive, inert cryofluid and has been the coolant of 
choice for cryogenic operation of high heat load synchrotron radiation optics.) Lowering the temperature of silicon from room temperature to liquid-nitrogen temperatures improves the so-called figure of merit (FOM), $k / \alpha$, by over forty fold. A major contribution to this field developed at the APS was the use of internally cooled optics, i.e., optics with liquid nitrogen flowing through them rather than in a contact-cooling arrangement in which the liquid nitrogen flows through a heat sink that is in thermal contact with the optical component. The internally cooled approach provides a more effective method for the removal of the power deposited in the optic than does the contact-cooling approach, thereby keeping the optic at an overall lower temperature. The primary technical difficulty to overcome when using this approach is development of a vacuum-tight seal between the coolant manifold and the crystal optical component that is radiation hard, thermally cyclable, and introduces minimal strains into the crystal, which would degrade the performance of the component. This problem is exacerbated by the fact that we desired the diffracting crystal to be thin (less that one millimeter) so that a large fraction of the incident beam would be transmitted, hence reducing the power to be removed by the liquid nitrogen. (Reducing the absorbed power is an inportant consideration because the operating temperature range for single-phase operation with liquid nitrogen is only a few degrees Centigrade, after which boiling could occur, a situation to be avoided.) These problems were overcome with a special crystal design [6] (see Figure 1) in combination with an indium-coated c-ring vacuum seal and special mounting design [7]. A crystal design was orginally tested at the ESRF under a collaborative agreement between the APS, ESRF, and SPring8 [7], and recently tested on the Synchrotron Radiation Instrumentation (SRI) Collaborative Access Team (CAT) insertion device beamline (Sector 1 ) at the APS. A summary of the results of the APS tests is shown in Figures 2 and 3 . As can be seen in Figure 2, very little broadening of the Si (111) rocking curve can be observed while even at the highest power densities, the rocking curve of the Si (333) reflection remained approximately constant at $2-3$ arc seconds, that being the residual strain in the thin part of the crystal from mounting and/or fabrication. Data in Figure 3 , taken not on the thin portion of the crystal but on the top of the thick portion, indicate that the thick portion of the crystal also performed well under thermal load. In fact, the Si (333) reflection is nearly at the theoretical value in the latter geometry, indicating both the thermal strain and mounting/fabrication strains are at the arc second level [8]. However it should be pointed out that these tests were conducted with a commissioning filter/Be window assembly upstream of the monochromator that removed nearly all of the low energy photons (a reduction of the total power density by about $12 \%$ with the undulator gap 
near fully closed, i.e., at $11.1 \mathrm{~mm}$ ). It is not yet clear whether this additional power will make any difference in the performance when using the thick portion of the monochromator, however the rate of liquid nitrogen use certainly increases when using the thick portion, and futher work will be required to look into such issues. Also planned are experiments with the commissioning window assembly removed, measurements of the beam brilliance from the monochromator system, and tests of a reliquefying system for the secondary liquid-nitrogen cooling loop that has recently been procured so that the entire monochromator/pump/reliquefier is a stand-alone system. Additional technical details on the APS cryogenic cooling program can be found in References 9 and 10.

The second monochromator design capable of withstanding the thermal loads generated by the APS insertion devices is the use of single crystal diamonds. Single-crystal diamond has approximately the same figure of merit at room temperature as silicon at liquid-nitrogen temperature (see Table I), but has the added advantage that it has a lower atomic number, and therefore absorbs considerably less power than

\section{Table 1}

\begin{tabular}{lllc} 
material & $\begin{array}{l}\mathrm{k}-\text { thermal } \\
\text { conductivity }\end{array}$ & $\begin{array}{l}\alpha-\text { coef. of } \\
\text { thermal expansion }\end{array}$ & $\begin{array}{c}|\mathrm{k} / \alpha| \\
\mathrm{FOM}\end{array}$ \\
\hline Si $\left(300^{\circ} \mathrm{K}\right)$ & $1.48 \mathrm{~W} / \mathrm{cm}-{ }^{\circ} \mathrm{C}$ & $2.3 \times 10^{-6} /{ }^{\circ} \mathrm{K}$ & 1 \\
Si $\left(80^{\circ} \mathrm{K}\right)$ & $13.4 \mathrm{~W} / \mathrm{cm}-{ }^{\circ} \mathrm{C}$ & $-0.5 \times 10^{-6} /{ }^{\circ} \mathrm{K}$ & 42 \\
Diamond $\left(300^{\circ} \mathrm{K}\right)$ & $15-20 \mathrm{~W} / \mathrm{cm}-{ }^{\circ} \mathrm{C}$ & $0.8 \times 10^{-6} /{ }^{\circ} \mathrm{K}$ & $29-39$
\end{tabular}

silicon for a given thickness. The disadvantage of diamond, besides the obvious one of obtaining large perfect single crystals, is that the integrated reflectivity of the (111) diamond reflection is about half that of silicon (111). (For an overview of the diffraction properties of diamond see ref. 11. ) Nonetheless, because diamonds do not require the complicated cryogenic cooling systems of silicon monochromators and can be used in tandem (the transmitted beam can be used as the incident beam for other monochromators futher downstream), they are an appealing option for some users of third-generation sources. Recently we have undertaken tests of a double-crystal monochromator, where both the first 
and second crystals are high quality synthetic diamonds. Figure 4 shows the mounting scheme employed for the first crystal of the double-diamond monochromator. Good thermal contact between the diamond and the watercooled substrate is ensured by a thin layer of liquid gallium/indium alloy between the diamond and substrate, a technique used a decade ago for silicon crystals [12] and successfully adapted for use with diamonds at the ESRF [2]. The liquid metal not only provides for good thermal contact but also holds the diamond on the substrate though surface tension. Figure 5 is a summary of the results. Although encouraging from a thermal point of view, the quality of the data is not nearly as good as that from the silicon. This is due in part to the fact that the diamonds were not perfect, and results were dependent on the location of the $x$-ray beam on the diamond. Comparisons of the flux from the diamond and silicon indicate that the estimate of about a factor of two decrease in flux in the diamond, as compared to silicon, is indeed the case [13].

\section{Milli-eV Resolution In-Line Monochromators}

High energy resolution monochromators are a critical ingredient in state-of-the-art inelastic scattering and nuclear resonant scattering beamlines. In these photon hungry experiments, it is necessary to reduce the beam with a bandwidth of a few $\mathrm{eV}$ from the high heat load monochromator to the few milli-eV level with very high efficiency. This can be achieved by designing optics to accept as much of the incident beam divergence as possible while simultaneously limiting the energy acceptance to the narrow bandwidth required. A variety of approaches to produce milli-eV beams have been attempted, including $(+,-,-,+)$ dispersive crystal arrangements [14] and scattering at Bragg angles very near $90^{\circ}$ [15]. The former approach has suffered from loss of throughput because of the mismatch between the angular acceptance of the optics and the angular divergence of the x-ray beam, while the latter operate at only discrete $x$-ray energies that satisfy Bragg's law in the back-scattering geometry for some (hkl) reflection. Ishikawa et al. [16], in 1992, proposed a new approach, the nested channel cut, that would produce a milli-eV resolution beam with a high throughput at any energy by manipulating the angular divergence of the beam and angular acceptance of the crystal. An asymmetric geometry on the first reflection of the "outer channel-cut" crystal increases the acceptance angle (over that of a symmetrical geometry) and provides a better match for the incident beam divergence while simultaneously providing a highly collimated diffracted beam on the output side to match the angular acceptance of the first reflection of the "inner channel-cut" crystal. The "inner channel cut" then selects the 
desired energy width. The second reflection from each of the two channel cuts then replicates the incident beam direction and vertical phase space (i.e., size and divergence). The operation of the device is best seen by looking at the corresponding Dumond diagram shown in Figure 6 . Very good efficiencies are expected from such an arrangement, especially when used on a high brilliance source, such as the APS. Using this phase space manipulation concept, several nested channel-cut, high resolution monochromators have been designed, fabricated, and tested on Sector 3 of the APS [17]. Figure 7 shows a schematic of a nested channel-cut monochromator that was developed to operate near $14.4 \mathrm{keV}$ for the nuclear resonant scattering program. Initial test results have been very promising (See Flg. 8). A beam with resolution of 5 milli-eV has been obtained on Sector 3 with excellent throughput. Very recently a beam with a FWHM of less that 1 milli-eV has been produced using a slight variation on this approach [17a].

\section{Dual Polarization, High Energy X-ray Phase Plates}

The production and manipulation of $\mathrm{x}$-ray polarization states by crystal optics has been an ongoing program at the APS. It is our belief that the use of crystal optics for the production of highly polarized hard $x$-ray beams not only can generate beams with a higher degree of circular polarization than specialized insertion devices but offers opportunities of greater flexibility at a substantially lower cost. The primary motivation for the production of high energy circularly polarized $x$-ray beams is for magnetic Compton scattering, although they may have applications in elastic scattering experiments as well. High energy circularly polarized $\mathrm{x}$-rays (or any other polarization state for that matter) can be generated by taking advantage of the slight differences in the sigma and pi wavevectors that occur near the Bragg condition when scattering in the Laue or transmission geometry. If the normal to the atomic scattering planes is oriented at $45^{\circ}$ with respect to the (linear) polarization vector of the incident radiation, then sigma and pi wavefields will exist within the crystal with equal amplitudes. For a fixed thickness Laue crystal, a $(2 n-1) \pi / 2$ phase shift between the sigma and pi wavefields (of a given branch of the dispersion surface) will occur for specific $x$-ray energies, and circularly polarized $x$-rays can be generated. Because the beta branch of the dispersion curve has a $180^{\circ}$ phase shift relative to the alpha branch, if both branches are allowed to propagate through the crystal and recombine at the exit surface, the effect will be nullified. If, however, the material and thickness is chosen such that only the wavefields associated with the alpha branch survive, then the polarization of the 
output beam can be manipulated. The value of the phase shift varies over the width of the rocking curve, so the more collimated the beam, the better the performance is expected of the phase plate. It is for this reason, that these phase plates should perform much better with highly collimated beams, such as those produced by undulators on thirdgeneration sources, than on bending magnet or wiggler beamlines. The first attempts at producing high energy ( $>50 \mathrm{keV}$ ) circularly polarized $x-$ rays using this approach were made using a silicon phase plate [18], but it was realized that a higher atomic number material would be more effective (to completely absorb the $\beta$-branch of the dispersion curve) and germanium plates were developed that proved to provide a much higher degree of circular polarization [19].

In the case of magnetic Compton scattering experiments, to extract the magnetic portion of the Compton profile from the very much larger electronic component, switching of the direction of the spins in the sample or reversal of the handedness of the circular polarization is required. Spin-direction reversal works well with relatively soft ferromagnetic samples, but such switching is not possible with all materials and/or experimental geometries. Therefore sources that can provide polarized beams of both handedness are very desirable. Although it was in principal possible to change the handedness of the radiation in the first generation of high energy phase-plates, this process was not totally satisfactory. A method for improved helicity reversal was suggested by Hirano et al. [20] but suffered from a limited throughput. In the latest design developed at the APS, the Hirano concept has been extended to retain a high throughput by simultaneously producing two circularly polaraized radiation beams, displaced in space from one another, of opposite handedness. A schematic of this new phase plate is shown in Figure 9. The Bragg reflection serves to monochromate the incident (filtered) white beam and to act a beam splitter sending two monochromatic beams (from the $\langle 110\rangle$ and $\langle 1-10\rangle$ family of planes) to the equilavnet planes in the Laue geometry, where the phase shifting is accomplished to produce the circularly polarized $x$-rays. If the plane containing the $\langle 1-10\rangle$ and $\langle 110\rangle$ reciprocal lattice vectors is normal to the plane of diffraction from the $<100>$ planes, then a condition for multiple diffraction is satisfied for all three reflections $(\langle 1-10\rangle,\langle 110\rangle$, and $<100>$ ), a condition that can alter the desired polarization effect. The multiple-beam condition can be eliminated by rotating about the $<100\rangle$ vector, which breaks the symmetry of the geometry. (The angles between the incident beam and the two sets of $\{110\}$-type planes are no longer equal, and the energies of the $\langle 1-10\rangle$ and $\langle 110\rangle$ reflections occur at slightly different values.) The $x$-ray polarization incident on the sample 
is changed by a small translation (millimeters) of the sample from one beam to the other.

This device was recently tested on the Sector 1 undulator beamline of SRI CAT. The degree of circular polarization was measured on one of the beams at various energies and on both beams at an energy where the degree of circular polarization was calculated to be near unity $(P c= \pm 1)$. The results of these measurements are shown in Figure 10. The high degree of polarization of both handedness that can be produced with this new type of phase plate will open up magnetic Compton scattering to a host of new magnetic materials in which switching of the spin direction is impossible.

\section{Phase Zone Plates}

The use of zone plates for the focusing of x-rays is well established in the synchrotron community, especially in the soft $x$-ray microscopy community [21,22]. A zone plate is a structure composed of alternating concentric rings of two materials wih different (complex) refractive indices. The focusing capability is based on constructive interference of the wavefront modified by passage through the zone plate. The wavefront modification is obtained through the introduction of a relative change in amplitude or phase in the beams emerging from two neighboring zones. A zone plate is called an amplitude zone plate if the focusing results from the different absorption between two neighboring zones. It is called a phase zone plate if a phase change is the mechanism for the focusing. If illuminated with an x-ray beam whose spatial coherence length is equal to or greater than the size of the zone plate, a diffraction-limited focus can be obtained. Third-generation sources, such as the APS, can provide the required spatial coherence at appreciable flux levels. (Temporal coherence is also required but only to the extend that $E / \Delta E$ is greater than the number of rings or zones.) The size of the focal spot from the zone plate is determined by the width of the outermost ring. Using state-ofthe art lithographic technologies, zones plates with outermost ring widths of less than 100 nanometers can currently be fabricated.

In the soft $x$-ray region, zone plate efficiency is limited to about $15 \%$ due to photoelectric absorption. For $x$-ray energies greater than 3 $\mathrm{keV}$, it is in principle possible to produce phase zone plates with focusing efficiencies close to $40 \%$. In a recent experiment on the Sector 2 insertion device beamline at the APS with the zone plate placed behind a Si (111) monochromator (bandwidth about $0.01 \%$ ), a focal spot size (FWHM) of 0.46 
microns in the vertical direction and 1.2 microns in the horizontal direction was obtained at $11.4 \mathrm{keV}$ as in Figure 11. A photon flux of $4 \times 10^{9}$ photons/s was obtained at the focus with the storage ring operating at $40 \mathrm{~mA}$ [23].

\section{Conclusions}

Single-crystal optics have been the mainstay of synchrotron radiation optical components in the past and will continue to be important at third-generation sources. Although silicon is certainly the workhorse of single crystals, as pointed out in this paper, good quality diamonds and germanium can play important roles. All of the single-crystal optical components described above were fabricated and/or initially characterized off-line in the APS X-ray Optics Fabrication and Characterization Facility. The importance of such a facility in the development of these and other x-ray optics components cannot be overstated. During the development of the APS and it $s$ associated support facilities, provisions were included for an $x$-ray single-crystal fabrication and characterization laboratory [24], a deposition facility, and a metrology laboratory [25]. All three of these facilities are now up and running at the APS and provide invaluable support to researchers developing and testing $x$-ray optical components.

In the case of the zone plate, it is the synthesis of two unrelated fields that have come together to make such a device practical, namely synthethic or man-made x-ray optical components and the use of coherent $x$-rays. We believe that man-made components will play an ever increasing role in the future of $\mathrm{x}$-ray optics development. Not only will high quality and high speed deposition systems be required for mirror, multilayer, and zone-plate-based optics, but hard x-ray lithographic techniques will become an important tool for the fabrication of new $x$-ray optical components. In addition to the deposition facility mentioned above, the SRI CAT has included a program of deep-etch $x$-ray lithography to be based on the bending magnet beamline of Sector 2 specifically for the development of such optical components.

In the early planning stages of third-generation synchrotron radiation sources, there were serious concerns as to whether the full brilliance of these sources could ever be brought to the experiment because of thermal degradation of the first optical components. Within the current operational envelope of the two working, third-generation hard $x$-ray storage rings (ESRF and APS), the intitial indications are that 
this will not be the case (although, at this point in time, few careful measurements of beam brilliance after the first high heat load components have been made and published). Delivery of a high brilliance monochromatic beam to downstream optical components permits novel devices, such as milli-eV resolution monochromators and phase zone plates, to work to their full potential. However, the combined effects of increases in stored beam current, longer insertion devices, and smaller magnetic gaps will provide a continuing challenge to scientists and engineers working on high heat load optical components to provide high brilliance monochromatic beams to new and innovative $x$-ray optical devices that will surely be developed at these unique third-generation sources.

\section{Acknowledgments}

The work described in this overview is due to the effort of many staff members of the Experimental Facilities Division of the Advanced Photon Source. The cryogenically cooled silicon and diamond monochromator experimental team included Shawn Rogers, Patricia Fernandez, Wah-Keat Lee, Tim Graber, and Lahsen Assoufid. Work on the in-line nested monochromator described above was undertaken by Ercan Alp, Tom Toellner, Tim Mooney, Wolfgang Sturhahn, and Klaus Quast. The phase plate work was performed by Wenbing Yun, B. Lai, Z. Cai, D. Legnini, E. Gluskin, A. Krasnoperova, Z. Chen, F. Cerinna, E, DiFabrizio, and M. Centili. Contributors to the development and testing of the high energy $x-$ ray phase plate were Jonathan Lang, George Srajer, Sarjvit Shastri, Dean Haeffner, and Christie Nelson. Thanks also go to lan McNulty for clear explanations on the operations of zone plates. All of the silicon and germanium crystal optical components were fabricated in the APS Optics Fabrication and Characterization lab, run by Felix Krasnicki. We also acknowledge the Harris Diamond Corporation for working with the APS in obtaining the high quality diamonds used in these tests. This work was supported by the U.S. Department of Energy, BES Materials Sciences, under contract no. W-31-109-ENG-38. 


\section{References}

1. Andreas K. Freund, SPIE Vol. 2515, 445, 1995.

2. Andreas K Freund, Opt. Eng., 34, 432, 1995.

3. Gerard Marot, Opt. Eng., 34, 426, 1995.

4. Victor Rehn, SPIE Vol. 582, 238, 1985.

5. D. H. Bilderback, Nuc. Instrum. and Meths. A246, 434, 1986.

6. G. S. Knapp, C. S. Rogers, M. A. Beno, G. Jennings, and P. L. Cowan, Rev. Sci. Instrum. 66, 2138, (1995).

7. C. S. Rogers, D. M. Mills, P. B. Fernandez, G. S. Knapp, M. Wulff, M. Handfland, M. Rossat, A. Freund, G. Marot, J. Holmberg, and H. Yamaoka, "Further Tests on Liquid-nitrogen-cooled, Thin Siliconcrystal Monochromators using a Focused Wiggler Synchrotron Beam," accepted for publication in Rev. Sci. Instrum.

8. C. S. Rogers, D. M. Mills, W.-K. Lee, P. B. Fernandez, and T. Graber, "Experimental results with cryogencially cooled.thin, silicon crystal $x$-ray monochromators on high-heat-flux beamlines", presented at the SPIE Meeting, Aug. 4-9, 1996, Denver, CO.

9. C. Shawn Rogers, D. M. Mills and L. Assoufid, "The Cryogenic Cooling Program at the Advanced Photon Source," Argonne National Laboratory Report ANL/APS/TB-18, June 1994.

10. D. M. Mills, "Cryogenically Cooled Monochromators for the Advanced Photon Source," accepted for publication in Rev. Sci. Instrum.

11. R. C. Blasdell, L. A. Assoufid, and D. M. Mills, "Diamond Monochromators for APS Undulator-A Beamlines," Argonne National Laboratory, ANL/APS/TB-24, September 1995.

12. D. H. Bilderback, D. M. Mills, B. W. Batterman, and C. Henderson, Nuc. Instrum. and Meths. A246, 428, 1986.

13. Patricia Fernandez and Wah-Keat Lee, APS, ANL , private communication, 1996. 
14. G. Faigel, D. P. Siddons, J. B. Hastings, P. E. Haustein, J. R. Grover, J. P. Remeika, and A. S. Cooper, Phys. Rev. Lett. 58, 2699, 1987.

15. W. Graeff and G. Materlik, Nuc. Instrum. and Meths. 195, 97, 1982.

16. Tetsuya Ishikawa, Yoshitaka Yoda, Koichi Izumi, Carlos Kenichi Suzuki, Xiao Wei Zhang, Masami Ando, and Seishi Kituta, Rev. Sci. Instrum. 63, 1015, 1992.

17. T. M. Mooney, T. Toellner, W. Sturhahn, E.E. Alp, and S. D. Shastri, Nuc. Instrum. and Meths. A347, 348, 1994.

17a. Tom Toellner and Ercan Alp, APS, ANL, private communication, 1996.

18. D. M. Mills, Nuc. Inst. and Meths., A266, p531, 1988.

Note there are two corrections to this paper; Eqn. (3) should

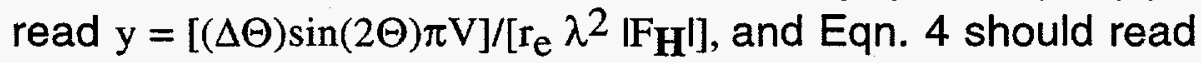
$Z_{\pi, \sigma}=\left[\left(\mathrm{r}_{\mathrm{e}} \lambda\left|\mathrm{F}_{\mathbf{H}}\right| \mathrm{PI} \mid\right) /(2 \pi \mathrm{V})\right]\left[\left(\mathrm{y}^{2}+1\right)^{1 / 2}-\left(\mathrm{y}^{2}+\cos (2 \Theta)\right)^{1 / 2}\right]$.

19. C. J. Yahnke, G. Srajer, D. R. Haeffner. D. M. Mills, and L. Assoufid, Nuc. Instrum. Meths, A347, 128, 1994.

20. H. Hirano, T. Ishikawa, I. Nakamura, M. Mozutani, and S. Kikuta, Jpn. J. Appl. Phys. 33, L689, 1994.

21. Janos Kirz, Chris Jacobsen, Malcolm Howeels, "Soft X-ray Microscopes and Their Biological Applications, "Quarterly Reviews of Biophysics, 28, 33-130, 1995.

22. B. Lai, W. Yun, D. Legnini, Y. Xiao, J. Chrzas, P. J. Viccaro, V. White, S. Bajikar, D. Denton, and F. Cerrina, "Hard x-ray phase zone plate fabricated by lithographic techniques", Appl. Phys. Lett. 61, $1877,1992$.

23. Wenbing Yun, APS, ANL, private communication, 1996.

24. S. Krasnicki "The APS Optics Topography Station," accepted for publication in Rev. Sci. Instrum.

25. C. Bresloff and D. M. Mills, "The Advanced Photon Source Metrology 
Lab," accepted for publication in Rev. Sci. Instrum. 


\section{Figure Captions}

Figure 1 Schematic design and cut-away view of the cryogenically cooled silicon crystal. The end view (a) shows the coolant channels and slot, the base of which serves as the diffracting surface for $\mathbf{x}$-rays. The slot has material removed from the underside creating a thin web as shown in (b). This thin web permits a portion of the incident beam to be transmitted, thereby reducing the total power absorbed in the crystal (c).

Figure 2 Experimental results for the thin cryo-cooled silicon crystal. The measured FWHM of the rocking curves as a function of the x-ray energy diffracted from the $\mathrm{Si}$ (111) planes is plotted (squares) along with the solid line representing the theoretical values. Data less than $9 \mathrm{keV}$ were taken on the first harmonic of the undulator while data collected above $10 \mathrm{keV}$ was taken using the third harmonic. No data were taken below $6 \mathrm{keV}$ due to absorption in the commissioning filter/window assembly. Also shown is the FWHM of the Si (333) relection (taken simultaneously with the $\mathrm{Si}$ (111) data) plotted as a function of the $\mathrm{x}$-ray energy from the (111) planes (diamonds). Hence the energy of the $x$-rays scattered from the (333) planes is three times the displayed absissa values. The near-constant value of about 1.5 arc seconds for the (333) width is indicative of residual strains in the system due to mounting and/or fabrication. The data were collected at stored beam currents between 50 and $60 \mathrm{~mA}$. Data collected at higher currents showed similar results.

Figure 3 Experimental results for the thick cryo-cooled silicon crystal. The measured FWHM of the rocking curves as a function of the x-ray energy diffracted from the Si (111) planes is plotted (squares) along with the solid line representing the theoretical values. Also shown is the FWHM of the Si (333) reflection (taken simultaneously with the Si (111) data) plotted as a function of the x-ray energy from the (111) planes, (diamonds). The energy of the $x$-rays scattered from the (333) planes is three times the displayed absissa values. Data less than $9 \mathrm{keV}$ were taken on the first harmonic of the undulator while data collected above $10 \mathrm{keV}$ were taken using the third harmonic. At about $9.6 \mathrm{keV}$, the tuning curves for the first and third harmonics of the undulator cross, and here data were taken at both the 1 st and 3rd harmonics. The results are identical for the (111) reflection but in the (333) data at $9.6 \mathrm{keV}$ you can see that one of the data points is higher, corresponding to the closed undulator gap (9.6 keV in the third harmonic), and a lower point corresponds to the open 
undulator gap ( $9.6 \mathrm{keV}$ in the first harmonic). No data were taken below 6 $\mathrm{keV}$ due to absorption in the commissioning filter/window assembly.

Figure 4 Diamond mounting scheme. The support for the synthetic diamond is a nickel-plated, water-cooled copper heat sink with a slot though which the power transmitted by the diamond can pass. A thin layer of liquid $\mathrm{In} / \mathrm{Ga}$ between the diamond and the cooled heat sink provides both good thermal contact of the diamond to the heat sink and a strain-free method of attaching the diamond to the heat sink (via the surface tension of the $\ln / G a$ alloy). The copper heat sink is plated with nickel to prevent the gallium from attacking the copper.

Figure 5 Experimental results for the diamond crystal. The measured FWHM of the rocking curves as a function of the x-ray energy diffracted from the (111) planes of diamond is plotted along with the solid line representing the theoretical values. Also shown is the FWHM of the (333) diamond reflection (taken simultaneously with the Si (111) data) plotted as a function of the $x$-ray energy from the (111) planes. Hence the energy of the $x$-rays scattered from the (333) planes is three times the displayed absissa values. Due to the slightly imperfect nature of the diamonds, the experimentally determined FWHM is slightly larger than theory. The spread in the data is primarly due to diffraction from different areas of the diamonds and not to thermal effects. For example, the (333) datum point at $9.6 \mathrm{kev}$ with a FWHM of about 2.5 arc seconds was collected at a value of the current twice that of the (333) datum with a FWHM of 3.8 arc seconds

Figure 6 The Dumond diagram for a nested crystal monochromator. The 442 crystal is asymmetrically cut to accept the full incident beam divergence (bold lines), as indicated by the nearly vertical lines in the center of the diagram. The output of the 442 reflection is shown with the acceptance of the (10 6 4) planes (dashed lines) near the zero on the $\Delta \Theta$ axis, the overlap indicated by the shaded area. For clarity, the overlap area and the Darwin width of the (10 6 4) have also been mapped onto the (422) input.

Figure 7 Schematic of nested crystal monochromator showing the asymmetrically cut (422) outer channel-cut and the symetrically cut (10 6 4) inner channel cut.

Figure 8 Experimentally determined resolution of the nested crystal monochromator tested on Sector 3. The output flux of the nested monochromator is plotted as a function of energy by keeping the outer channel cut fixed and rotating the inner channel cut. 
Figure 9 Schematic of the dual polarization phase plate. The entire device consists of a monolithic Bragg/Laue crystal pair for improved stability required for operation at high energies. The Bragg crystal serves as a monochromator and beam splitter, while the polarization manipulation is done in the Laue crystal. The two output beams, separated by several millimeters, have opposite helicities for specific $x$-ray energies.

Figure 10 Experimental results of dual beam phase plate. The measured and theoretical degree of circular polarization, Pc, measured as a function of energy for the (220) reflection (circles and solid line) and the (2-20) reflection (square and dashed line) for a $14.5-\mathrm{mm}$-thick germanium phase plate. The maxima (minima) at about $85 \mathrm{keV}(60 \mathrm{keV})$ corresponds to a phase shift of $\pi / 2(3 \pi / 2)$. Pc was determined experimentally via magnetic Compton scattering from iron.

Figure 11 Photograph of a phase zone plate used at the APS. 


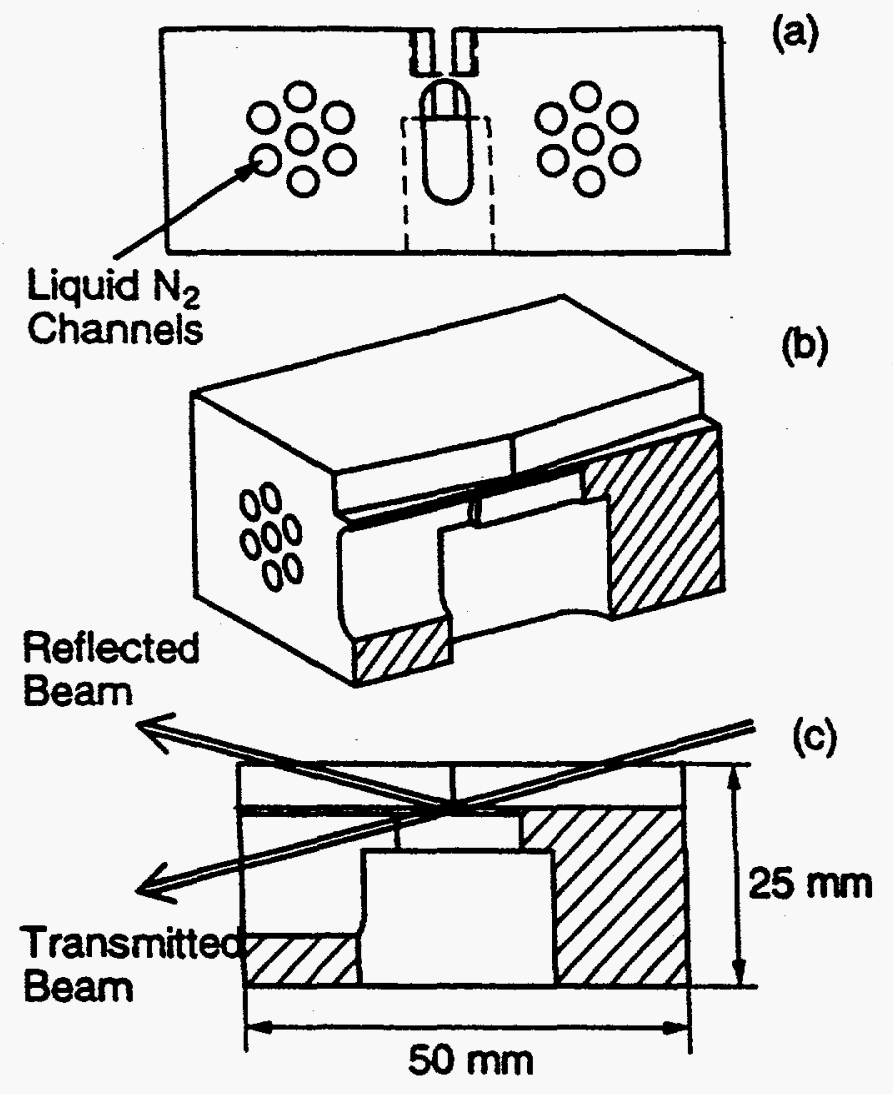

Fig 1 
Thin Silicon Crystal Energy and Gap Matched 50 to $60 \mathrm{~mA}$
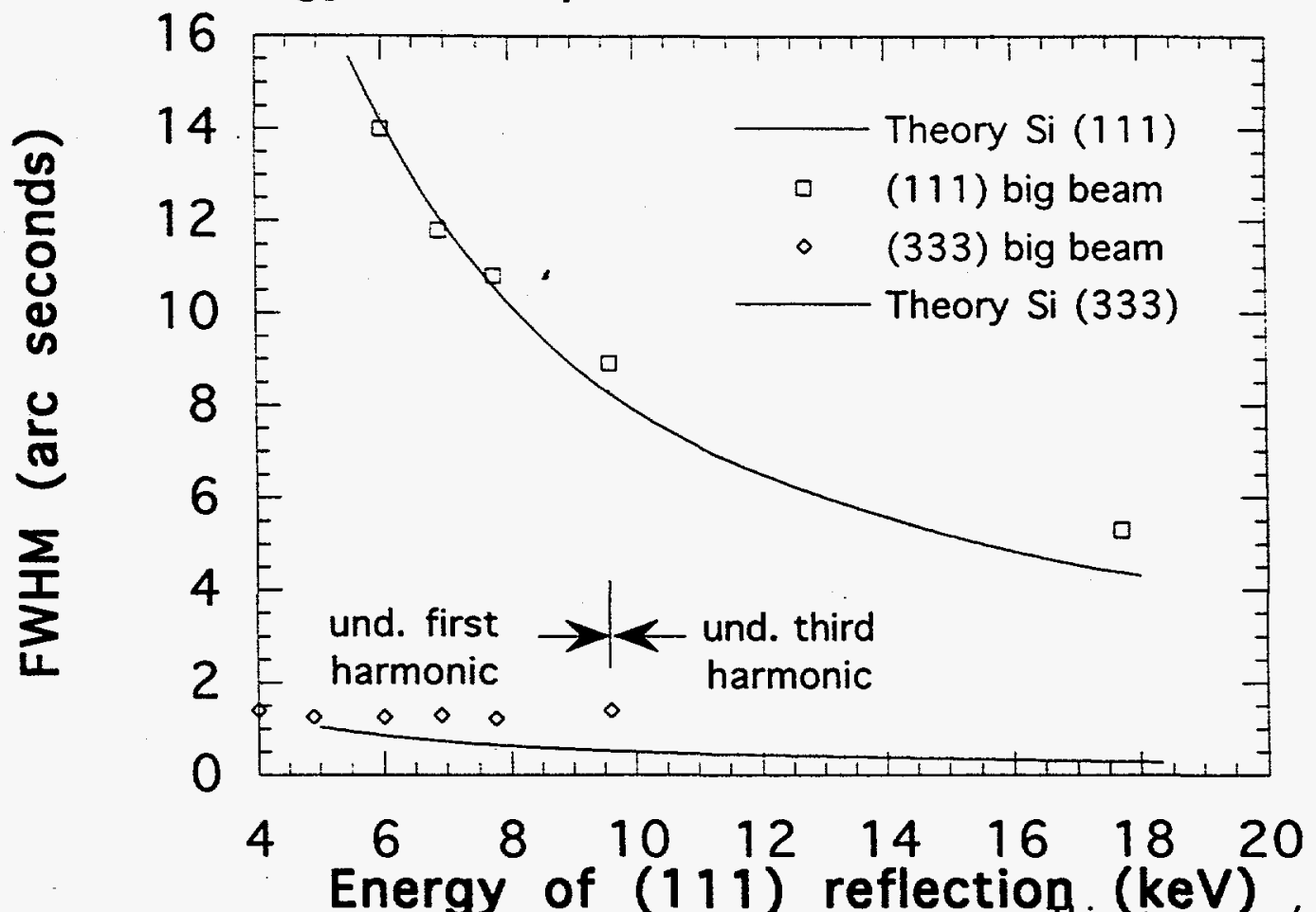

Thick Silicon Crystal Energy and Gap Matched 70 to $90 \mathrm{~mA}$

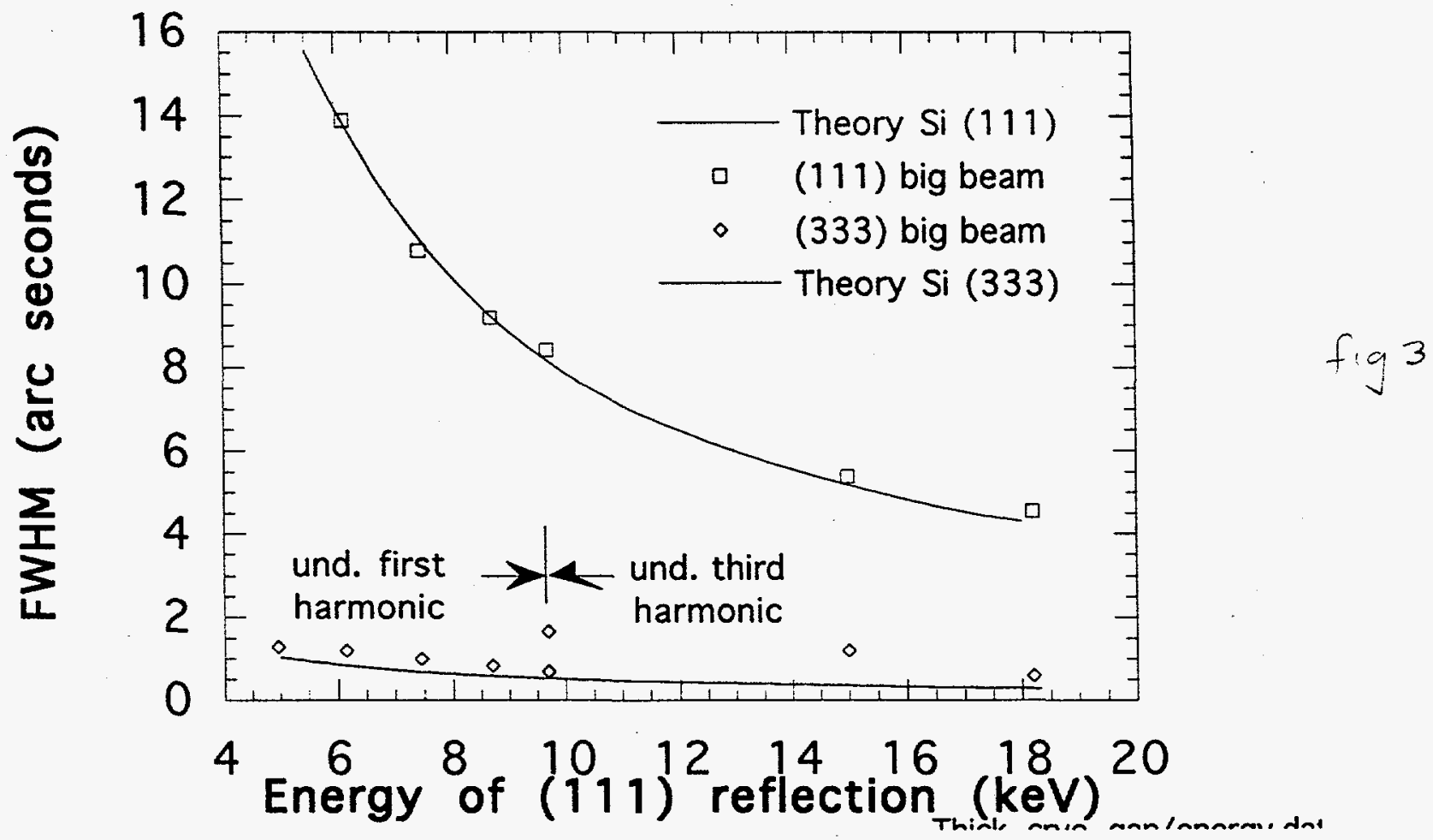


TOP VIEW
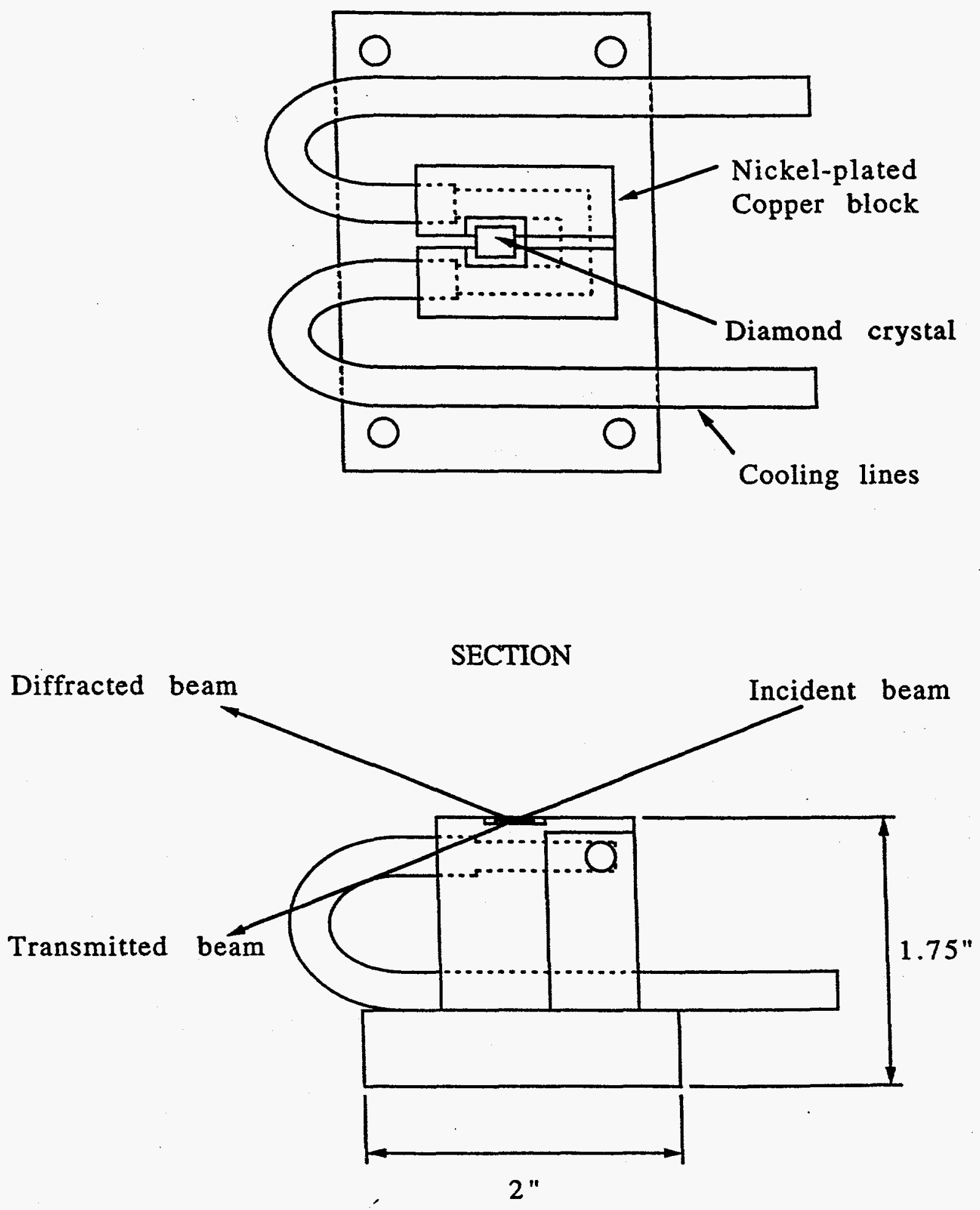


\section{Diamond Double Crystal Monochromator}

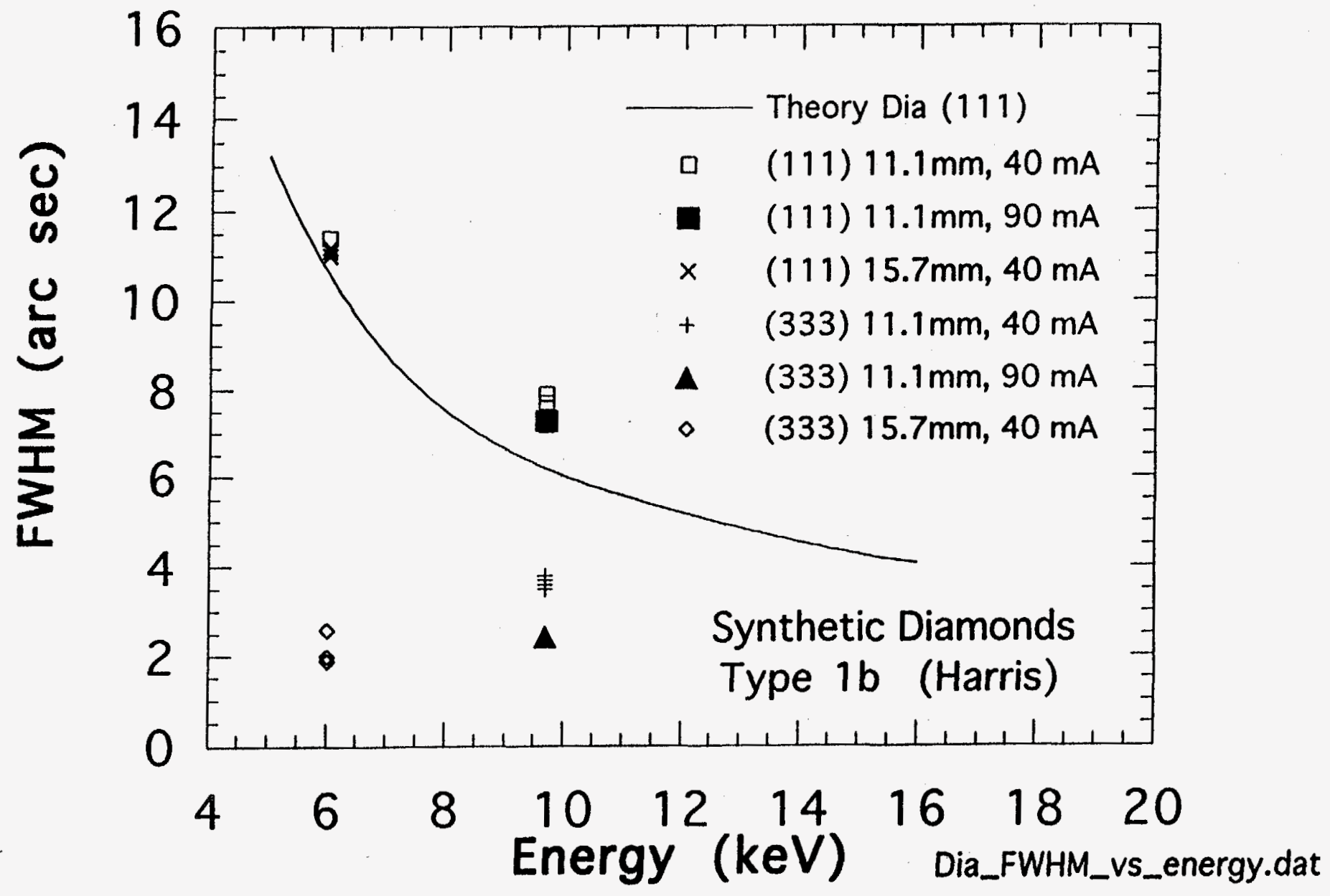


$\frac{9}{4}$

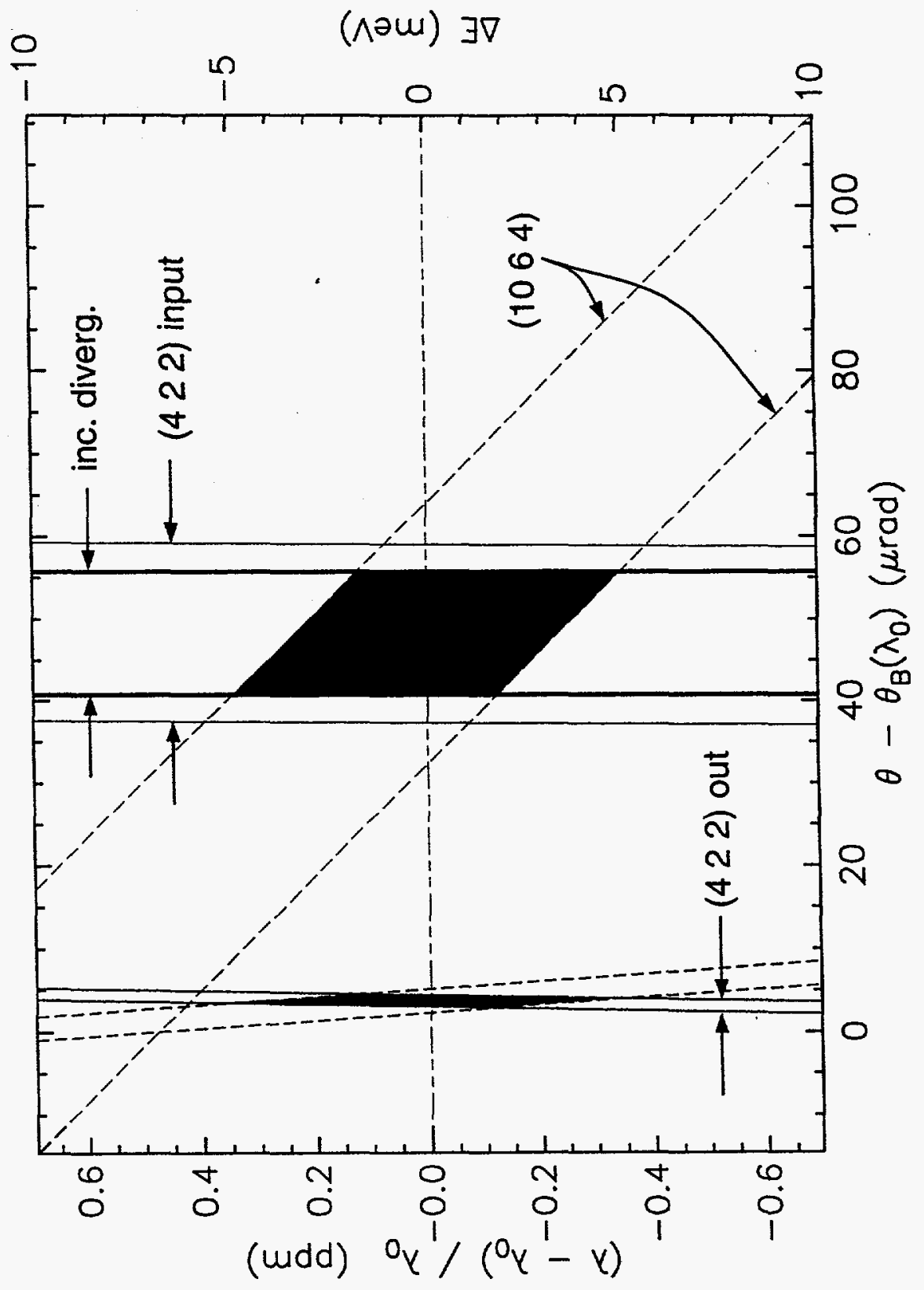




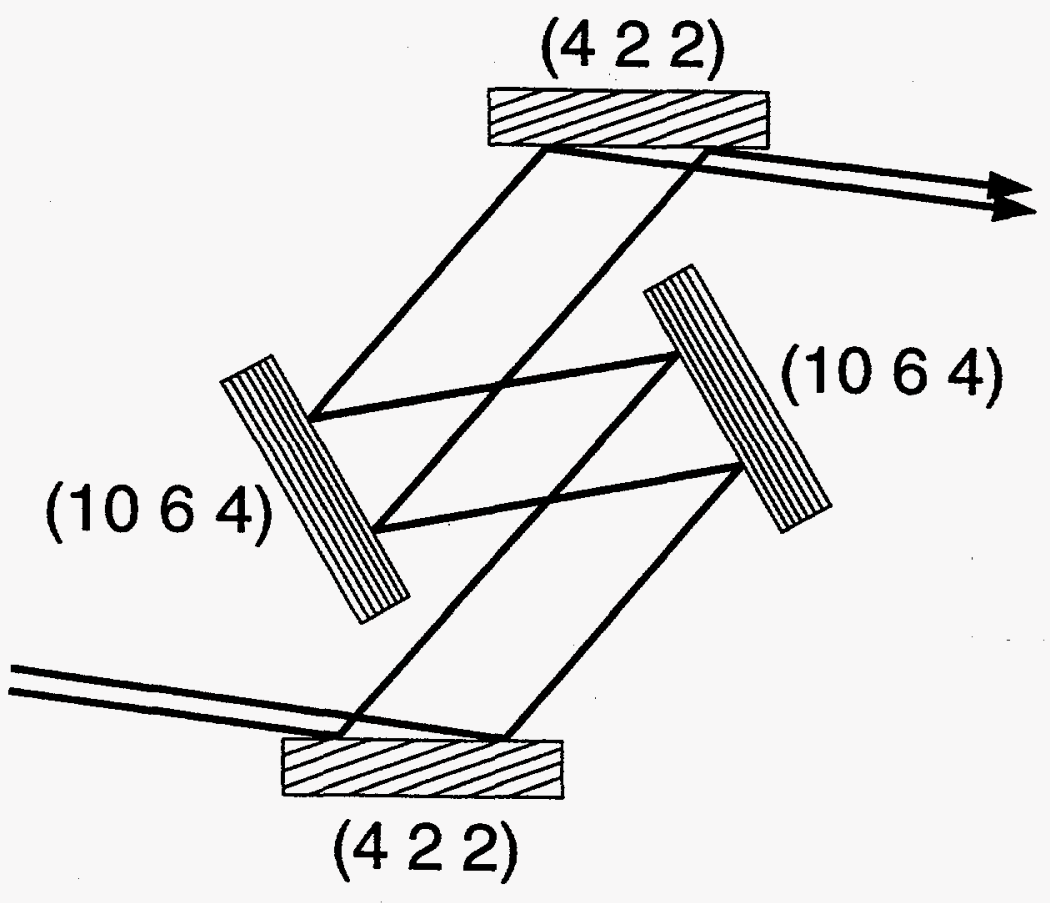

fig 7 


\section{ADVANCED PRIOTON SOUREE ZZZL}

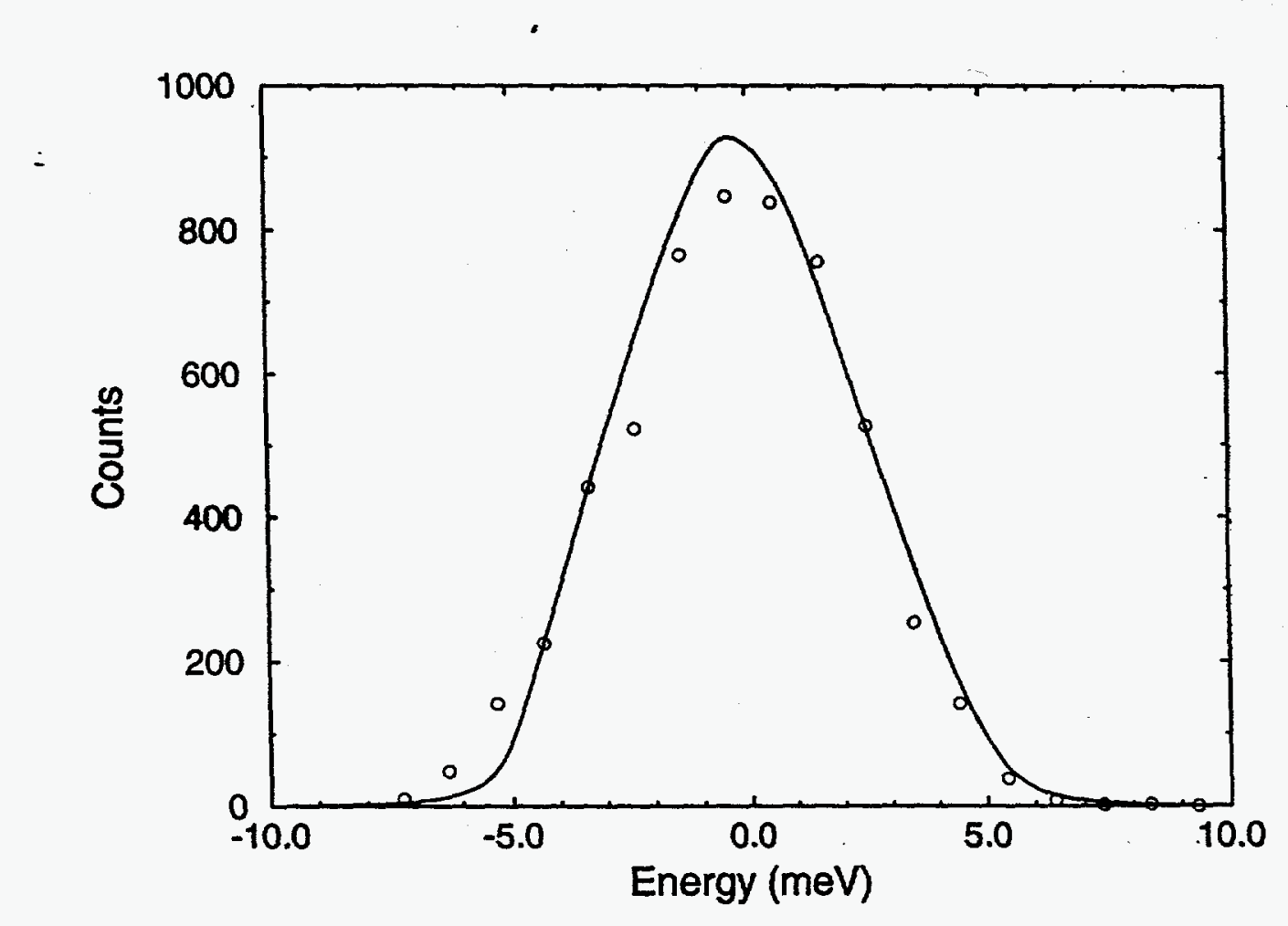

fig 8 

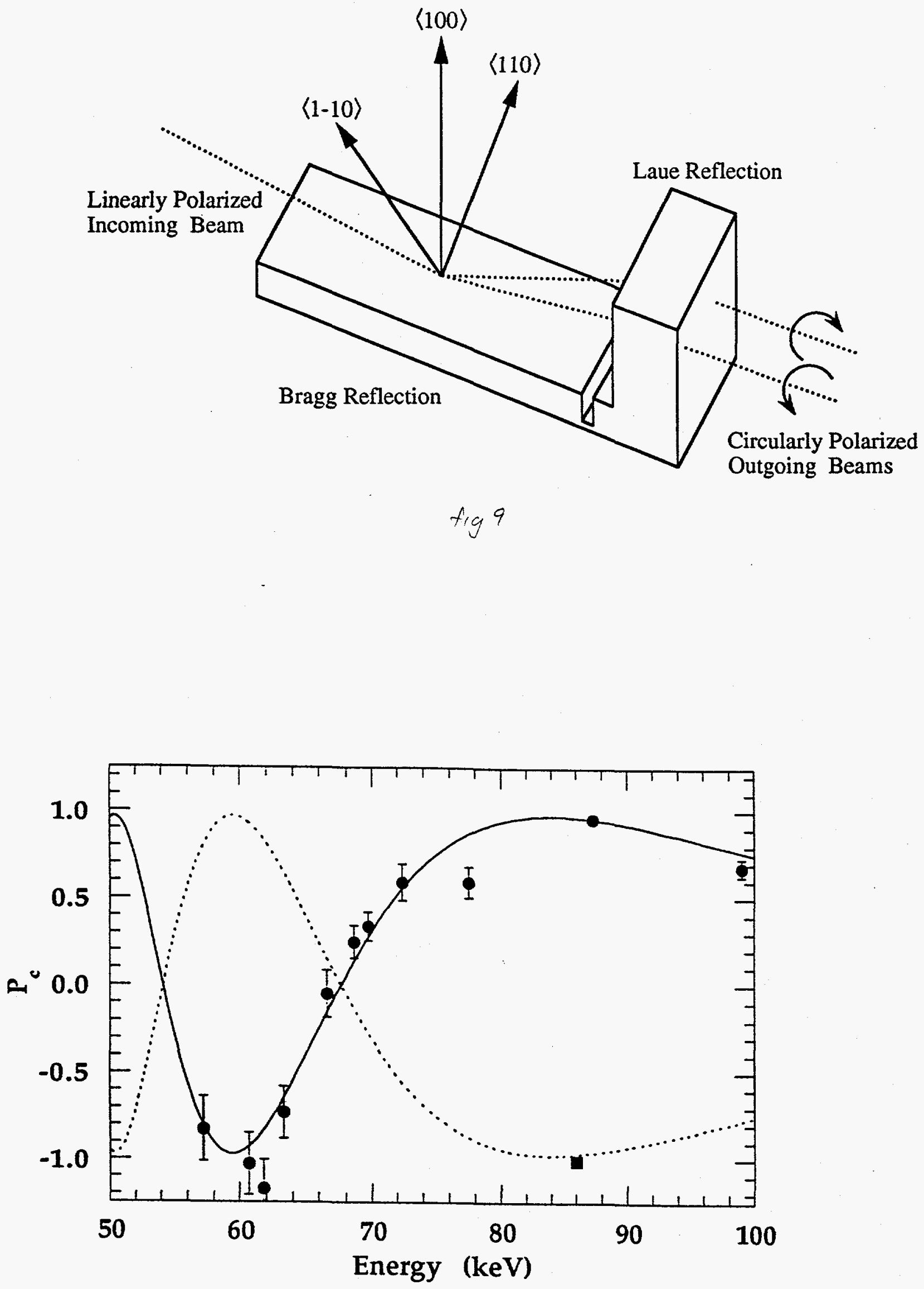


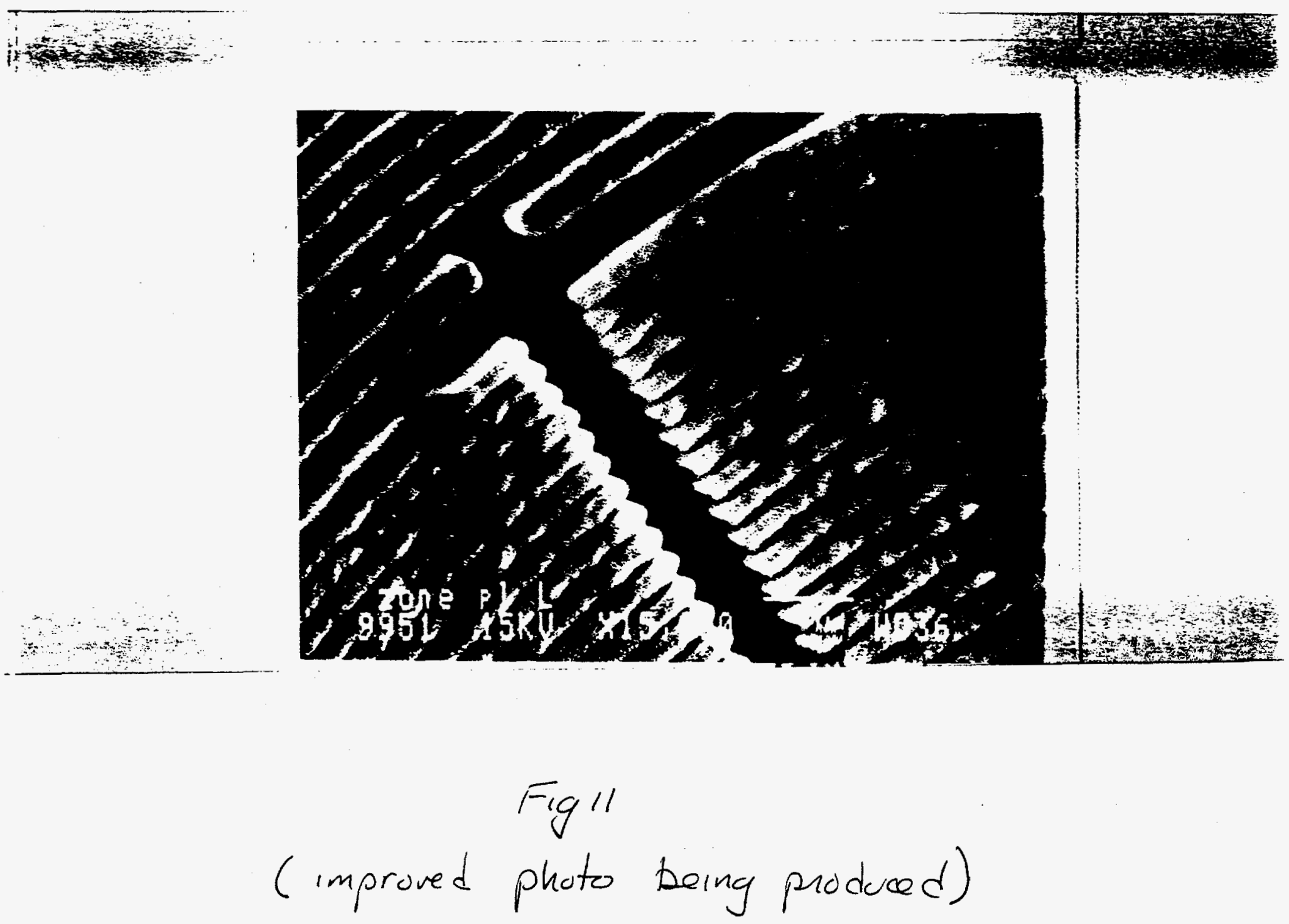

